\title{
LATTICE-BOLTZMANN TYPE RELAXATION SYSTEMS AND HIGH ORDER RELAXATION SCHEMES FOR THE INCOMPRESSIBLE NAVIER-STOKES EQUATIONS
}

\author{
MAPUNDI BANDA, AXEL KLAR, LORENZO PARESCHI, AND MOHAMMED SEAÏD
}

\begin{abstract}
A relaxation system based on a Lattice-Boltzmann type discrete velocity model is considered in the low Mach number limit. A third order relaxation scheme is developed working uniformly for all ranges of the mean free path and Mach number. In the incompressible Navier-Stokes limit the scheme reduces to an explicit high order finite difference scheme for the incompressible Navier-Stokes equations based on nonoscillatory upwind discretization. Numerical results and comparisons with other approaches are presented for several test cases in one and two space dimensions.
\end{abstract}

\section{INTRODUCTION}

Many kinetic equations or discrete velocity models of kinetic equations yield, in the limit for small Knudsen and Mach numbers, an approximation of the Incompressible Navier-Stokes (INS) equations. A classical example is given by the discrete velocity models used for Lattice-Boltzmann methods; see [6, 9, 19, 10, 8]. These discrete velocity models can be viewed as relaxation systems for the INS equations.

Relaxation type schemes have been used successfully to discretize such relaxation systems. In particular, a large number of numerical methods for kinetic equations with stiff relaxation terms have been considered in fluid dynamics or diffusive limits. For these relaxation methods and asymptotic-preserving methods, we refer to 11, 17, 26, 23, 24, 25, 28, and for more general applications of relaxation schemes we refer to the recent review paper [18. We mention here that, in the context of hyperbolic conservation laws relaxation schemes are closely related to central schemes 14, 37, 1, 22, 34, 31, 30, 41, in the sense that both approaches provide efficient high resolution and Riemann solver free numerical methods for hyperbolic conservation laws. Applications of central schemes to problems with stiff sources have been considered in 35, 15.

The aim of the present paper is to present a methodology for developing computational schemes for INS based on an appropriate discretization of a so-called

Received by the editor November 10, 2005 and, in revised form, January 15, 2007.

2000 Mathematics Subject Classification. Primary 76P05, 76D05, 65M06, 35B25.

Key words and phrases. Lattice-Boltzmann method, relaxation schemes, low Mach number limit, incompressible Navier-Stokes equations, high order upwind schemes, Runge-Kutta methods, stiff equations.

This work was supported by DFG grant KL 1105/9-1 and partially by TMR project "Asymptotic Methods in Kinetic Theory", Contract Number ERB FMRX CT97 0157.

(C)2007 American Mathematical Society Reverts to public domain 28 years from publication 
relaxation system; see Section 2 for more details of such a system. Such a relaxation system is derived from a Lattice-Boltzmann type discrete velocity model with diffuse scaling. The analytical derivations also demonstrate a relationship between the Lattice-Boltzmann method and such relaxation-based schemes. On the other hand one may refer to [30, 31, 32, 33], and references therein, for alternative approaches based on Godunov type methods.

In the present paper a third order relaxation scheme is developed. The scheme works with uniform accuracy with respect to the Knudsen and Mach numbers, and in the low Mach number limit it reduces to a third order explicit scheme for the INS equations. This is achieved by combining the ideas developed in 29, 24, 25 with third order nonoscillatory spatial discretizations and IMEX Runge-Kutta time discretizations [4, 38]. The high order nonoscillatory upwind method for the convective part of the relaxation system turns, in the INS limit (the relaxed scheme), into a high order treatment of the nonlinear convective parts of the INS equations. Clearly, to obtain only a discretization of the limit INS equations, one can use the above mentioned spatial discretization on the relaxation system and apply any high order time discretization directly to the resulting semi-discrete relaxed schemes. This allows us to obtain high order INS solvers with better stability properties.

The rest of the paper is organized in the following way. Section 2 contains the Lattice-Boltzmann type discrete velocity model and its equivalent associated closed moment system relaxing to the INS equations. Some simplified relaxation systems are also presented. In particular we introduce a simplified relaxation system that is suitable to provide relaxed schemes for the incompressible Navier-Stokes equations. Section 3 describes the time and space discretizations and includes a discussion of the discretization of the limit equations that originate from the schemes. Finally, Section 4 contains a numerical investigation of the schemes and a comparison with several different approaches in one and two space dimensions.

\section{LATTICE-BOLtZMANn TYPE DISCRETE VELOCITY MODELS AND SIMPLIFIED RELAXATION SYSTEMS}

2.1. The Lattice-Boltzmann moment system. The two-dimensional kinetic equation

$$
\frac{\partial f}{\partial t}+\mathbf{v} \cdot \nabla f=J(f)
$$

describes the evolution of a particle density $f(\mathbf{x}, \mathbf{v}, t)$ with $\mathbf{x}=(x, y) \in \mathbb{R}^{2}$ and $\mathbf{v}=\left(v_{1}, v_{2}\right) \in \mathbb{R}^{2}$. The left hand side of (1) represents free transport of the particles, while the right hand side describes interactions through collisions. For discrete models in $2 \mathrm{D}$ we have

$$
\mathbf{v} \in\left\{\mathbf{c}_{0}, \ldots, \mathbf{c}_{N-1}\right\}, \quad \mathbf{c}_{i} \in \mathbb{R}^{2}
$$

Here we consider a model with nine velocities $(N=9)$

$$
\begin{array}{llll}
\mathbf{c}_{1}=\left(\begin{array}{l}
1 \\
0
\end{array}\right), & \mathbf{c}_{2}=\left(\begin{array}{c}
0 \\
1
\end{array}\right), & \mathbf{c}_{3}=\left(\begin{array}{c}
-1 \\
0
\end{array}\right), & \mathbf{c}_{4}=\left(\begin{array}{c}
0 \\
-1
\end{array}\right), \\
\mathbf{c}_{5}=\left(\begin{array}{l}
1 \\
1
\end{array}\right), & \mathbf{c}_{6}=\left(\begin{array}{c}
-1 \\
1
\end{array}\right), & \mathbf{c}_{7}=\left(\begin{array}{c}
-1 \\
-1
\end{array}\right), & \mathbf{c}_{8}=\left(\begin{array}{c}
1 \\
-1
\end{array}\right),
\end{array}
$$

and $\mathbf{c}_{0}=\mathbf{0}$. In the discrete case, the $\mathbf{v}$-dependence of the particle distribution $f(\mathbf{x}, \mathbf{v}, t)$ is uniquely determined through $N$ functions

$$
f_{i}(\mathbf{x}, t)=f\left(\mathbf{x}, \mathbf{c}_{i}, t\right), \quad i=0, \ldots, N-1 .
$$


Macroscopic quantities like mass-, momentum- or energy-density are obtained by taking velocity moments of $f$. If $\zeta$ is any $\mathbf{v}$-dependent function, we denote the discrete velocity integral by

$$
\langle\zeta\rangle=\sum_{i=0}^{N-1} \zeta\left(\mathbf{c}_{i}\right) .
$$

Mass and momentum density are then given by

$$
\rho(\mathbf{x}, t)=\langle f(\mathbf{x}, \mathbf{v}, t)\rangle \quad \text { and } \quad \rho \mathbf{u}(\mathbf{x}, t)=\langle\mathbf{v} f(\mathbf{x}, \mathbf{v}, t)\rangle .
$$

In the following we denote the components of the velocity by $\mathbf{u}=\left(u_{1}, u_{2}\right)$. In Lattice-Boltzmann applications, the collision operator $J(f)$ in (11) is typically of BGK-type

$$
J(f)=-\frac{1}{\tau}\left(f-f^{e q}\right) .
$$

The parameter $\tau>0$ is called relaxation time and $f^{e q}$ is the equilibrium distribution. In the isothermal case, $f^{e q}$ depends on $f$ through the parameters $\rho$ and $\mathbf{u}$ which are calculated according to (2); see for example [19, 20, 42. For the standard D2Q9-model [40] with 9 velocities, we have

$$
f^{e q}[\rho, \mathbf{u}](\mathbf{v})=\rho\left(1+3 \mathbf{u} \cdot \mathbf{v}-\frac{3}{2}|\mathbf{u}|^{2}+\frac{9}{2}(\mathbf{u} \cdot \mathbf{v})^{2}\right) f^{*}(\mathbf{v})
$$

where $f^{*}$ is defined by

$$
f^{*}\left(\mathbf{c}_{i}\right)= \begin{cases}\frac{4}{9}, & i=0 \\ \frac{1}{9}, & i=1, \ldots, 4 \\ \frac{1}{36}, & i=5, \ldots, 8\end{cases}
$$

The equilibrium distribution is constructed in such a way that

$$
\langle J(f)\rangle=0 \quad \text { and } \quad\langle\mathbf{v} J(f)\rangle=0,
$$

which reflects conservation of mass and momentum in the collision process.

In order to obtain a relation between the kinetic equation (11) and the incompressible Navier-Stokes system, we introduce the diffusive scaling $\mathbf{x} \rightarrow \mathbf{x} / \epsilon, t \rightarrow t / \epsilon^{2}$ together with a rescaling of velocity $\mathbf{u} \rightarrow \epsilon \mathbf{u}$. This scaling describes the small Knudsen and low Mach number limit of kinetic equations; see [4, 12, 5, 21, 1, for details. Under these transformations, (11) turns into

$$
\frac{\partial f}{\partial t}+\frac{1}{\epsilon} \mathbf{v} \cdot \nabla f=-\frac{1}{\epsilon^{2} \tau}\left(f-f^{e q}[\rho, \epsilon \mathbf{u}]\right) .
$$

In our case, (4) consists of nine equations for the occupation numbers $f_{0}, \ldots, f_{8}$. In order to get closer in notation to the Navier-Stokes system, we transform (4) into an equivalent set of moment equations (see also [29, 13] for a similar approach) 
using moments based on the following v-polynomials [17:

$$
\begin{array}{lll}
P_{0}(\mathbf{v})=1 & \\
P_{1}(\mathbf{v})=\frac{v_{1}}{\epsilon}, & P_{2}(\mathbf{v})=\frac{v_{2}}{\epsilon}, \\
P_{3}(\mathbf{v})=\frac{v_{1}^{2}}{\epsilon^{2}}-\frac{1}{3 \epsilon^{2}}, & P_{4}(\mathbf{v})=\frac{v_{1} v_{2}}{\epsilon^{2}}, & P_{5}(\mathbf{v})=\frac{v_{2}^{2}}{\epsilon^{2}}-\frac{1}{3 \epsilon^{2}}, \\
P_{6}(\mathbf{v})=\frac{\left(3|\mathbf{v}|^{2}-4\right) v_{1}}{\epsilon^{3}}, & P_{7}(\mathbf{v})=\frac{\left(3|\mathbf{v}|^{2}-4\right) v_{2}}{\epsilon^{3}}, \\
P_{8}(\mathbf{v})=\frac{9|\mathbf{v}|^{4}-15|\mathbf{v}|^{2}+2}{\epsilon^{4}} . & &
\end{array}
$$

Note that $\left\langle P_{0} f\right\rangle=\rho,\left\langle P_{1} f\right\rangle=\rho u_{1}$ and $\left\langle P_{2} f\right\rangle=\rho u_{2}$. The second order moments form a symmetric tensor

$$
\boldsymbol{\Theta}=\left(\boldsymbol{\Theta}^{x}, \boldsymbol{\Theta}^{y}\right)=\left(\begin{array}{ll}
\theta_{11} & \theta_{12} \\
\theta_{12} & \theta_{22}
\end{array}\right)=\left(\begin{array}{ll}
\left\langle P_{3} f\right\rangle & \left\langle P_{4} f\right\rangle \\
\left\langle P_{4} f\right\rangle & \left\langle P_{5} f\right\rangle
\end{array}\right),
$$

where

$$
\mathbf{\Theta}^{x}=\left(\begin{array}{c}
\theta_{11} \\
\theta_{12}
\end{array}\right), \quad \boldsymbol{\Theta}^{y}=\left(\begin{array}{c}
\theta_{12} \\
\theta_{22}
\end{array}\right),
$$

and for the remaining moments we set

$$
\mathbf{q}=\left(\begin{array}{l}
q_{1} \\
q_{2}
\end{array}\right)=\left(\begin{array}{c}
\left\langle P_{6} f\right\rangle \\
\left\langle P_{7} f\right\rangle
\end{array}\right), \quad s=\left\langle P_{8} f\right\rangle
$$

The equations of mass and momentum conservation are

$$
\begin{gathered}
\partial_{t} \rho+\operatorname{div} \rho \mathbf{u}=0, \\
\partial_{t} \rho \mathbf{u}+\operatorname{div} \Theta+\frac{1}{3 \epsilon^{2}} \nabla \rho=0 .
\end{gathered}
$$

Here, the divergence is applied to the rows of $\boldsymbol{\Theta}$. The equation for $\boldsymbol{\Theta}$ is

$$
\partial_{t} \boldsymbol{\Theta}+\frac{2}{3 \epsilon^{2}} \mathbf{S}[\rho \mathbf{u}]+\frac{1}{3} \mathbf{Q}[\mathbf{q}]=-\frac{1}{\epsilon^{2} \tau}(\boldsymbol{\Theta}-\rho \mathbf{u} \otimes \mathbf{u}),
$$

where

$$
\mathbf{S}[\mathbf{u}]=\frac{1}{2}\left(\begin{array}{cc}
2 \partial_{x} u_{1} & \partial_{y} u_{1}+\partial_{x} u_{2} \\
\partial_{y} u_{1}+\partial_{x} u_{2} & 2 \partial_{y} u_{2}
\end{array}\right)
$$

and

$$
\mathbf{Q}[\mathbf{q}]=\left(\begin{array}{cc}
\partial_{y} q_{2} & \partial_{y} q_{1}+\partial_{x} q_{2} \\
\partial_{y} q_{1}+\partial_{x} q_{2} & \partial_{x} q_{1}
\end{array}\right) .
$$

Finally, the third and fourth order moments satisfy

$$
\begin{gathered}
\partial_{t} \mathbf{q}+\frac{1}{\epsilon^{2}} \operatorname{div}\left(\begin{array}{cc}
\theta_{22} & 2 \theta_{12} \\
2 \theta_{12} & \theta_{11}
\end{array}\right)+\frac{1}{6} \nabla s=-\frac{1}{\epsilon^{2} \tau} \mathbf{q}, \\
\partial_{t} s+\frac{4}{\epsilon^{2}} \operatorname{div} \mathbf{q}=-\frac{1}{\epsilon^{2} \tau} s .
\end{gathered}
$$

Altogether, we obtain a hyperbolic system with stiff relaxation terms. The determination of the diffusion limit of the above system is straightforward. From the momentum equation in (5) we conclude that $\nabla \rho$ tends to zero as $\epsilon \rightarrow 0$. Hence, $\rho$ 
approaches a constant $\bar{\rho}$ (which is the Boussinesq relation in the isothermal case). Writing $\rho=\bar{\rho}\left(1+3 \epsilon^{2} p\right)$, equation (5) transforms into

$$
\begin{gathered}
\partial_{t} p+\frac{1}{3 \epsilon^{2}} \operatorname{div} \mathbf{u}=-\operatorname{div}(p \mathbf{u}), \\
\partial_{t} \mathbf{u}+\operatorname{div} \frac{1}{\bar{\rho}} \boldsymbol{\Theta}+\nabla p=-3 \epsilon^{2} \partial_{t}(p \mathbf{u}) .
\end{gathered}
$$

For $\epsilon \rightarrow 0$, equation (6) yields at the lowest order

$$
\frac{1}{\bar{\rho}} \Theta=\mathbf{u} \otimes \mathbf{u}-\frac{2 \tau}{3} \mathbf{S}[\mathbf{u}] .
$$

Since (7) decouples completely from the other equations (in lowest order) and since $2 \operatorname{div} \mathbf{S}[\mathbf{u}]=(\Delta+\nabla \operatorname{div}) \mathbf{u}$, we obtain from (8) and (9) the incompressible NavierStokes equations as a limiting system

$$
\begin{gathered}
\operatorname{div} \mathbf{u}=0, \\
\partial_{t} \mathbf{u}+\operatorname{div} \mathbf{u} \otimes \mathbf{u}+\nabla p=\frac{\tau}{3} \Delta \mathbf{u},
\end{gathered}
$$

where the Reynolds number is related to the relaxation time by $R e=3 / \tau$.

We remark that (5), (6) and (7) can be viewed as a relaxation system for the Navier-Stokes equations (10).

2.2. Simplified relaxation systems. We consider the system of equations in (6) and (8). For numerical reasons we simplify this system in such a way that the limit as $\epsilon$ tends to zero is preserved, i.e. is the same as in the original system, (6) and (8).

From equation (8) we neglect the term $-\operatorname{div} p \mathbf{u}$ and $-3 \epsilon^{2} \partial_{t} p \mathbf{u}$. From equation (66) we neglect the term $\frac{1}{3} \mathbf{Q}[\mathbf{q}]$ and introduce a new term, $\nabla^{\mathbf{a}}[\mathbf{u}]$, as follows:

For $p, \mathbf{u}=\left(u_{1}, u_{2}\right)$ and $\boldsymbol{\Theta}=\left(\boldsymbol{\Theta}^{x}, \boldsymbol{\Theta}^{y}\right)=\left(\begin{array}{ll}\theta_{11} & \theta_{12} \\ \theta_{12} & \theta_{22}\end{array}\right)$ as defined above, we consider the system

$$
\begin{gathered}
\partial_{t} p+\frac{1}{\epsilon^{2}} \operatorname{div} \mathbf{u}=0, \\
\partial_{t} \mathbf{u}+\operatorname{div} \boldsymbol{\Theta}+\frac{1}{\bar{\rho}} \nabla p=0, \\
\partial_{t} \boldsymbol{\Theta}+\nabla^{\mathbf{a}}[\mathbf{u}]+\frac{2}{\epsilon^{2}} \mathbf{S}^{\epsilon}[\mathbf{u}]=-\frac{1}{\epsilon^{2} \tau}(\boldsymbol{\Theta}-\mathbf{u} \otimes \mathbf{u}),
\end{gathered}
$$

where

$$
\mathbf{S}^{\epsilon}[\mathbf{u}]=\mathbf{S}[\mathbf{u}]-\frac{\epsilon^{2}}{2} \nabla^{\mathbf{a}}[\mathbf{u}] .
$$

We have added and subtracted the term

$$
\nabla^{\mathbf{a}}[\mathbf{u}]=\left(a^{2} \partial_{x} \mathbf{u}, b^{2} \partial_{y} \mathbf{u}\right)=\left(\begin{array}{ll}
a^{2} \partial_{x} u_{1} & b^{2} \partial_{y} u_{1} \\
a^{2} \partial_{x} u_{2} & b^{2} \partial_{y} u_{2}
\end{array}\right)
$$

where $a^{2} \partial_{x} \mathbf{u}=\left(\begin{array}{l}a^{2} \partial_{x} u_{1} \\ a^{2} \partial_{x} u_{2}\end{array}\right)$ and $b^{2} \partial_{y} \mathbf{u}=\left(\begin{array}{l}b^{2} \partial_{y} u_{1} \\ b^{2} \partial_{y} u_{2}\end{array}\right)$ with $\mathbf{a}=\left(\begin{array}{l}a \\ b\end{array}\right) \in \mathbb{R}_{+}^{2}$. Obviously the limit equations for this system are again the incompressible Navier-Stokes equations with Reynolds number $R e=1 / \tau$. 
Remark 1. Considering the nonstiff advection parts in (11) separately for $\mathbf{u}$ and $\Theta$ we obtain a hyperbolic system with characteristic speeds $\pm a$ and $\pm b$ in $x$ and $y$ direction

$$
\begin{aligned}
& \partial_{t} \mathbf{u}+\operatorname{div} \boldsymbol{\Theta}=0, \\
& \partial_{t} \boldsymbol{\Theta}+\nabla^{\mathbf{a}}[\mathbf{u}]=0 .
\end{aligned}
$$

As we will see in section 4, $\mathbf{a}$ is chosen depending on the local speeds.

We can build a second relaxation system by neglecting the time derivative in the first equation of (11) without altering the other equations. This is practical if one considers an implementation in the vorticity formulation as given below. To this aim we consider the system

$$
\begin{gathered}
\operatorname{div} \mathbf{u}=0, \\
\partial_{t} \mathbf{u}+\operatorname{div} \boldsymbol{\Theta}+\frac{1}{\bar{\rho}} \nabla p=0, \\
\partial_{t} \boldsymbol{\Theta}+\nabla^{\mathbf{a}}[\mathbf{u}]=-\frac{1}{\epsilon^{2} \tau}\left(\boldsymbol{\Theta}-\mathbf{u} \otimes \mathbf{u}+2 \tau \mathbf{S}^{\epsilon}[\mathbf{u}]\right) .
\end{gathered}
$$

We introduce the vorticity $\omega=\partial_{x} u_{2}-\partial_{y} u_{1}$ by taking a two-dimensional curl of the second and third equation in (13) and applying the divergence-free condition, $\operatorname{div} \mathbf{u}=0$. A relaxation system for vorticity is then derived as

$$
\begin{aligned}
\partial_{t} \omega+\operatorname{div} \boldsymbol{\Phi} & =0 \\
\partial_{t} \boldsymbol{\Phi}+\nabla^{\mathbf{a}}[\omega] & =-\frac{1}{\epsilon^{2} \tau}(\boldsymbol{\Phi}-\omega \mathbf{u}+2 \tau \nabla \omega),
\end{aligned}
$$

where

$$
\omega \in \mathbb{R}, \quad \boldsymbol{\Phi}=\nabla \times \boldsymbol{\Theta}=\left(\begin{array}{l}
\varphi_{1} \\
\varphi_{2}
\end{array}\right) \in \mathbb{R}^{2}, \quad \mathbf{u}=\left(\begin{array}{c}
u_{1} \\
u_{2}
\end{array}\right) \in \mathbb{R}^{2}, \quad \nabla^{\mathbf{a}}[\omega]=\left(\begin{array}{c}
a^{2} \partial_{x} \omega \\
b^{2} \partial_{y} \omega
\end{array}\right) .
$$

For $\boldsymbol{\Theta}$ the curl is taken row-wise. Then $\mathbf{u} \in \mathbb{R}^{2}$ is determined by solving the Poisson problem

$$
\Delta \psi=\omega, \quad \mathbf{u}=\nabla^{\perp} \psi=\left(\begin{array}{c}
-\partial_{y} \psi \\
\partial_{x} \psi
\end{array}\right),
$$

with $\psi$ denoting the stream function. In the latter relaxation equations (13) the whole system reduces to only three equations (for the variables $\omega, \varphi_{1}, \varphi_{2}$ ) instead of six (for the variables $u_{1}, u_{2}, \theta_{11}, \theta_{12}, \theta_{21}, \theta_{22}$ ) as in the case of primitive variables.

Having developed the Lattice-Boltzmann type relaxation systems (equation (11), (13) or (14)), what remains is to develop high-order relaxation schemes which in the limit as $\epsilon \rightarrow 0$ converge uniformly to numerical schemes for incompressible Navier-Stokes equations. We will present a full discussion on some schemes that can be used to compute the relaxation systems in Section 3 below.

\section{NuMERICAL SCHEMES}

To develop numerical schemes for the relaxation systems developed in Section 2.2 above, we will consider equation (11). This equation is used as a case study for our derivations since to develop numerical schemes for the other systems, namely (13) and (14), one needs analogous manipulations and neglecting similar terms as presented above. 
3.1. Space discretizations. In this section high order upwind discretizations are developed for the nonstiff advection part in (11). The stiff part is treated by high order centered differences, as in 24, 25. In the remainder of this section the time continuous version of the scheme is considered (method of lines). The full spacetime discretization is obtained combining the spatial discretization obtained here with the time discretization described in the next subsection.

To discretize the equations in space we use a uniform grid in the $x$ - and $y$ directions with grid points $\left(x_{i}, y_{j}\right)$ with spacing $h$. Consider the nonstiff linear part of the system in equations (11) as presented in (12). One observes that for the $x$ direction $\Theta^{x} \pm a \mathbf{u}$ are the characteristic variables associated with the characteristic speeds $\pm a$. For the $y$-direction the characteristic variables associated with the characteristic speeds $\pm b$ are $\boldsymbol{\Theta}^{y} \pm b \mathbf{u}$. According to these considerations the values of the characteristic variables are determined at cell-boundaries following the approach in [26. This can be done in a straightforward way for a second order method. For a third order method we use for the reconstruction step a third order CWENO interpolant [1. Similar reconstructions were also applied in 2, 3. We report for the convenience of the reader the polynomials, $\mathbf{p}_{i j}(\mathbf{z} ; x)$, for the reconstruction in $2 \mathrm{D}$ in the $x$-direction. These polynomials, in the cell $(i, j)$ with cell-center $\left(x_{i}, y_{j}\right)$, in the case of a second order method, are given by

$$
\mathbf{p}_{i j}(\mathbf{z} ; x)=\mathbf{z}_{i j}+\mathbf{s}_{i j}\left(x-x_{i}\right),
$$

where the MinMod limiter in $\mathbf{s}_{i j}$,

$$
\mathbf{s}_{i j}(\mathbf{z})=\frac{1}{h} \operatorname{MinMod}\left(\mathbf{z}_{i j}-\mathbf{z}_{i-1 j}, \mathbf{z}_{i+1 j}-\mathbf{z}_{i j}\right),
$$

is applied componentwise. The variable $\mathbf{z}_{i j}$ denotes the cell average of a vector function $\mathbf{z}(x, y)$ in the cell $(i, j)$ taken from a set, $\mathbf{z}=\left\{\mathbf{z}_{i j}\right\}$, for all cells $(i, j)$ in the computational domain.

For the third order CWENO case using Simpson's rule we have

$$
\mathbf{p}_{i j}(\mathbf{z} ; x)=w_{L} \mathbf{P}_{i j}^{L}(\mathbf{z} ; x)+w_{R} \mathbf{P}_{i j}^{R}(\mathbf{z} ; x)+w_{C} \mathbf{P}_{i j}^{C}(\mathbf{z} ; x),
$$

with

$$
\begin{aligned}
& \mathbf{P}_{i j}^{R}(\mathbf{z} ; x)=\mathbf{z}_{i j}+\frac{1}{h}\left(\mathbf{z}_{i+1 j}-\mathbf{z}_{i j}\right)\left(x-x_{i}\right), \\
& \mathbf{P}_{i j}^{L}(\mathbf{z} ; x)=\mathbf{z}_{i j}+\frac{1}{h}\left(\mathbf{z}_{i j}-\mathbf{z}_{i-1 j}\right)\left(x-x_{i}\right),
\end{aligned}
$$

and

$$
\begin{aligned}
\mathbf{P}_{i j}^{C}(\mathbf{z} ; x)= & \mathbf{z}_{i j}-\frac{1}{12}\left(\mathbf{z}_{i+1 j}-2 \mathbf{z}_{i j}+\mathbf{z}_{i-1 j}\right)-\frac{1}{12}\left(\mathbf{z}_{i j+1}-2 \mathbf{z}_{i j}+\mathbf{z}_{i j-1}\right) \\
& +\frac{1}{2 h}\left(\mathbf{z}_{i+1 j}-\mathbf{z}_{i-1 j}\right)\left(x-x_{i}\right)+\frac{1}{h^{2}}\left(\mathbf{z}_{i+1 j}-2 \mathbf{z}_{i j}+\mathbf{z}_{i-1 j}\right)\left(x-x_{i}\right)^{2} .
\end{aligned}
$$

In expression (16) for $k=L, R, C$

$$
w_{k}=\frac{\alpha_{k}}{\sum_{l} \alpha_{l}}, \quad \alpha_{k}=\frac{c_{k}}{\left(\gamma+I S_{k}\right)^{\beta}},
$$


and

$$
\begin{aligned}
c_{L} & =\frac{1}{4}, \quad c_{R}=\frac{1}{4}, \quad c_{C}=\frac{1}{2}, \\
I S_{L} & =\left(v_{i j}-v_{i-1 j}\right)^{2}, \quad I S_{R}=\left(v_{i+1 j}-v_{i j}\right)^{2}, \\
I S_{C} & =\frac{13}{3}\left(v_{i+1 j}-2 v_{i j}+v_{i-1 j}\right)^{2}+\frac{1}{4}\left(v_{i+1 j}-v_{i-1 j}\right)^{2},
\end{aligned}
$$

with $\gamma=10^{-6}, \beta=2$. Clearly any other high order reconstruction procedure applies.

We proceed further with the MUSCL approach as in 26] to determine the characteristic variables at the boundary of the cells $\left[x_{i-1 / 2}, x_{i+1 / 2}\right]$

$$
\begin{gathered}
\left(\boldsymbol{\Theta}^{x}+a \mathbf{u}\right)_{i+1 / 2 j}=\mathbf{p}_{i j}\left(\boldsymbol{\Theta}^{x}+a \mathbf{u} ; x_{i+1 / 2}\right), \\
\left(\boldsymbol{\Theta}^{x}-a \mathbf{u}\right)_{i+1 / 2 j}=\mathbf{p}_{i+1 j}\left(\boldsymbol{\Theta}^{x}-a \mathbf{u} ; x_{i+1 / 2}\right) .
\end{gathered}
$$

An analogous procedure is used for the $y$ direction and $\left(\boldsymbol{\Theta}^{y} \pm b \mathbf{u}\right)_{i j+1 / 2}$.

We denote by $\mathbf{F}_{h}^{(1)}, \mathbf{F}_{h}^{(2)}$ the discretization of the convective parts $\operatorname{div} \boldsymbol{\Theta}$ and $\nabla^{\mathbf{a}}[\mathbf{u}]$ in equation (11), respectively. They are described as follows:

Using the reconstruction polynomial given above componentwise one obtains

$$
\mathbf{F}_{h}^{(1)}(\boldsymbol{\Theta}, \mathbf{u})=\frac{1}{h}\left(\boldsymbol{\Theta}_{i+1 / 2 j}^{x}-\Theta_{i-1 / 2 j}^{x}\right)+\frac{1}{h}\left(\Theta_{i j+1 / 2}^{y}-\Theta_{i j-1 / 2}^{y}\right),
$$

and

$$
\mathbf{F}_{h}^{(2)}(\boldsymbol{\Theta}, \mathbf{u})=\left(\frac{1}{h}\left(a^{2} \mathbf{u}_{i+1 / 2, j}-a^{2} \mathbf{u}_{i-1 / 2 j}\right), \frac{1}{h}\left(b^{2} \mathbf{u}_{i j+1 / 2}-b^{2} \mathbf{u}_{i j-1 / 2}\right)\right),
$$

where the numerical fluxes are given by

$$
\begin{aligned}
& \mathbf{\Theta}_{i+1 / 2 j}^{x}=\frac{1}{2}\left(\Theta_{i j}^{x}+\Theta_{i+1 j}^{x}\right)-\frac{a}{2}\left(\mathbf{u}_{i+1 j}-\mathbf{u}_{i j}\right) \\
& +\frac{1}{2}\left(\sigma_{i j}^{(1)}\left(\Theta^{x}+a \mathbf{u}\right)-\sigma_{i+1 j}^{(1)}\left(\Theta^{x}-a \mathbf{u}\right)\right), \\
& \boldsymbol{\Theta}_{i j+1 / 2}^{y}=\frac{1}{2}\left(\Theta_{i j}^{y}+\Theta_{i j+1}^{y}\right)-\frac{b}{2}\left(\mathbf{u}_{i j+1}-\mathbf{u}_{i j}\right) \\
& +\frac{1}{2}\left(\sigma_{i j}^{(2)}\left(\mathbf{\Theta}^{y}-b \mathbf{u}\right)-\sigma_{i j+1}^{(2)}\left(\mathbf{\Theta}^{y}-b \mathbf{u}\right)\right), \\
& \mathbf{u}_{i+1 / 2 j}=\frac{1}{2}\left(\mathbf{u}_{i j}+\mathbf{u}_{i+1 j}\right)-\frac{1}{2 a}\left(\Theta_{i+1 j}^{x}-\Theta_{i j}^{x}\right) \\
& +\frac{1}{2 a}\left(\sigma_{i j}^{(1)}\left(\Theta^{x}+a \mathbf{u}\right)+\sigma_{i+1 j}^{(1)}\left(\Theta^{x}-a \mathbf{u}\right)\right), \\
& \mathbf{u}_{i j+1 / 2}=\frac{1}{2}\left(\mathbf{u}_{i j}+\mathbf{u}_{i j+1}\right)-\frac{1}{2 b}\left(\Theta_{i j+1}^{y}-\Theta_{i j}^{y}\right) \\
& +\frac{1}{2 b}\left(\sigma_{i j}^{(2)}\left(\boldsymbol{\Theta}^{y}+b \mathbf{u}\right)+\sigma_{i j+1}^{(2)}\left(\mathbf{\Theta}^{y}+b \mathbf{u}\right)\right) .
\end{aligned}
$$

Here $a$ and $b$ can be chosen locally; see the last section for further details. In the second order case the $\sigma_{i j}^{(k)}, k=1,2$, are given by

$$
\sigma_{i j}^{(1)}(\mathbf{z})=\frac{1}{2} \operatorname{MinMod}\left(\mathbf{z}_{i j}-\mathbf{z}_{i-1 j}, \mathbf{z}_{i+1 j}-\mathbf{z}_{i j}\right),
$$

and

$$
\sigma_{i j}^{(2)}(\mathbf{z})=\frac{1}{2} \operatorname{MinMod}\left(\mathbf{z}_{i j}-\mathbf{z}_{i j-1}, \mathbf{z}_{i j+1}-\mathbf{z}_{i j}\right) .
$$


In the third order case the CWENO reconstruction gives

$$
\begin{aligned}
\sigma_{i j}^{(1)}(\mathbf{z})= & \frac{w_{R}}{2}\left(\mathbf{z}_{i+1 j}-\mathbf{z}_{i j}\right)+\frac{w_{L}}{2}\left(\mathbf{z}_{i j}-\mathbf{z}_{i-1 j}\right)+\frac{w_{C}}{4}\left(\mathbf{z}_{i+1 j}-\mathbf{z}_{i-1 j}\right) \\
& -\frac{w_{C}}{12}\left(\mathbf{z}_{i+1 j}-2 \mathbf{z}_{i j}+\mathbf{z}_{i-1 j}\right)-\frac{w_{C}}{12}\left(\mathbf{z}_{i j+1}-2 \mathbf{z}_{i j}+\mathbf{z}_{i j-1}\right) \\
& +\frac{w_{C}}{4}\left(\mathbf{z}_{i j+1}-2 \mathbf{z}_{i j}+\mathbf{z}_{i j-1}\right),
\end{aligned}
$$

and

$$
\begin{aligned}
\sigma_{i j}^{(2)}(\mathbf{z})= & \frac{w_{R}}{2}\left(\mathbf{z}_{i j+1}-\mathbf{z}_{i j}\right)+\frac{w_{L}}{2}\left(\mathbf{z}_{i j}-\mathbf{z}_{i j-1}\right)+\frac{w_{C}}{4}\left(\mathbf{z}_{i j+1}-\mathbf{z}_{i j-1}\right) \\
& -\frac{w_{C}}{12}\left(\mathbf{z}_{i j+1}-2 \mathbf{z}_{i j}+\mathbf{z}_{i j-1}\right)-\frac{w_{C}}{12}\left(\mathbf{z}_{i+1 j}-2 \mathbf{z}_{i j}+\mathbf{z}_{i-1 j}\right) \\
& +\frac{w_{C}}{4}\left(\mathbf{z}_{i+1 j}-2 \mathbf{z}_{i j}+\mathbf{z}_{i-1 j}\right) .
\end{aligned}
$$

Now we must discretize the pressure variable and the stiff parts, i.e. terms with the coefficient $\frac{1}{\epsilon^{2}}$, in equations (11). First, we denote the discrete gradient by $\mathbf{G}_{h}$ and the discrete divergence by $\mathbf{D}_{h}$. They are given by second or fourth order centered differences, respectively. $\mathbf{S}_{h}^{\epsilon}$ and $\mathbf{S}_{h}$ denote second or fourth order centered difference approximations of $\mathbf{S}^{\epsilon}$ and $\mathbf{S}$.

Finally, we obtain a high order spatial discretization for the moment system characterized by

$$
\begin{aligned}
\dot{p}+\frac{1}{\epsilon^{2}} \mathbf{D}_{h} \cdot \mathbf{u} & =0, \\
\dot{\mathbf{u}}+\mathbf{F}_{h}^{(1)}(\boldsymbol{\Theta}, \mathbf{u})+\mathbf{G}_{h} p & =0, \\
\dot{\boldsymbol{\Theta}}+\mathbf{F}_{h}^{(2)}(\boldsymbol{\Theta}, \mathbf{u})+\frac{2}{\epsilon^{2}} \mathbf{S}_{h}^{\epsilon}(\mathbf{u}) & =-\frac{1}{\epsilon^{2} \tau}(\boldsymbol{\Theta}-\mathbf{u} \otimes \mathbf{u}),
\end{aligned}
$$

or equivalently

$$
\begin{aligned}
\mathbf{D}_{h} \cdot \mathbf{G}_{h} p-2 \epsilon^{2} \ddot{p} & =-\mathbf{D}_{h} \cdot \mathbf{F}_{h}^{(1)}(\boldsymbol{\Theta}, \mathbf{u}), \\
\dot{\mathbf{u}}+\mathbf{F}_{h}^{(1)}(\boldsymbol{\Theta}, \mathbf{u})+\mathbf{G}_{h} p & =0, \\
\dot{\boldsymbol{\Theta}}+\mathbf{F}_{h}^{(2)}(\boldsymbol{\Theta}, \mathbf{u}) & =-\frac{1}{\epsilon^{2} \tau}\left(\boldsymbol{\Theta}-\mathbf{u} \otimes \mathbf{u}+2 \tau \mathbf{S}_{h}^{\epsilon}(\mathbf{u})\right) .
\end{aligned}
$$

A corresponding high order upwind based space discretization for the incompressible Navier-Stokes equations is obtained by considering the limit of the above discretization as $\epsilon \rightarrow 0$ :

$$
\begin{aligned}
\mathbf{D}_{h} \cdot \mathbf{G}_{h} p & =-\mathbf{D}_{h} \cdot \mathbf{F}_{h}^{(1)}(\mathbf{u}), \\
\dot{\mathbf{u}} & =-\mathbf{F}_{h}^{(1)}\left(\mathbf{u} \otimes \mathbf{u}-2 \tau \mathbf{S}_{h}(\mathbf{u}), \mathbf{u}\right)-\mathbf{G}_{h} p .
\end{aligned}
$$

3.2. Time discretizations. To treat only the limit equations $(\epsilon=0)$ we could use any explicit high order Runge-Kutta method combined with a Poisson solver and the limiting (relaxed) spatial discretization. The Poisson equation is in this case only used to determine the divergence-free velocities via $\nabla p$ and not to advance the pressure for one time step. For example, the maximum preserving schemes in [34, 43. can be used or the DUMKA scheme [36 or simply a suitable Runge Kutta method. Several numerical tests in this direction are presented in the last section.

We need to point out that it is necessary to be careful if an explicit time discretization is to be used. Due to the diffusion terms, standard explicit schemes 
would suffer from excessive time step restrictions since in that case $\nu \Delta t \leq c h^{2}$ for a suitable constant $c$ of order unity, which depends on the scheme. This is where DUMKA, as an explicit scheme, is advantageous to use since it has a large value of c; hence larger time steps are admissible.

Further, a more challenging issue is to obtain a discretization of the relaxation system that works uniformly for all ranges of the parameter $\epsilon$, thus allowing us to study the numerical passage from the discrete velocity models for the Boltzmann equation to the INS regime.

To this aim we can use implicit-explicit (IMEX) Runge-Kutta methods of the type developed in [4, 38. In these schemes the nonstiff parts are treated explicitly and the stiff ones are treated implicitly. In particular, we will treat the pressurevelocity coupling in an implicit way. As we will see, in the small mean free path limit $\epsilon \rightarrow 0$ this leads to a projection scheme for the incompressible Navier Stokes equations. First, second and third order time discretizations are discussed in the sequel.

We denote the time step by $k$ and use the superscript $n$ to denote the time iterations. For the first order method we can use the following simple time discretization:

$$
\begin{aligned}
\mathbf{u}^{n+1} & =\mathbf{u}^{n}-k\left(\operatorname{div} \boldsymbol{\Theta}^{n}+\nabla p^{n+1}\right) \\
\boldsymbol{\Theta}^{n+1} & =\boldsymbol{\Theta}^{n}-k \nabla^{\mathbf{a}}\left[\mathbf{u}^{n}\right]-\frac{k}{\epsilon^{2} \tau}\left(\boldsymbol{\Theta}^{n+1}+2 \tau \mathbf{S}^{\epsilon}\left[\mathbf{u}^{n+1}\right]-\mathbf{u}^{n+1} \otimes \mathbf{u}^{n+1}\right), \\
p^{n+1} & =p^{n}-\frac{k}{\epsilon^{2}} \operatorname{div} \mathbf{u}^{n+1} .
\end{aligned}
$$

Using the last equation in (18) into the first equation in (18) yields a Helmholtz equation for the pressure

$$
\Delta p^{n+1}-\frac{\epsilon^{2}}{k^{2}} p^{n+1}=\frac{1}{k} \operatorname{div} \mathbf{u}^{n}-\operatorname{div} \operatorname{div} \Theta^{n}-\frac{\epsilon^{2}}{k^{2}} p^{n} .
$$

This equation can be solved by a suitable iterative method. Then $\mathbf{u}^{n+1}$ is determined using the first equation in (18).

Obviously, as $\epsilon \rightarrow 0$ the time integration scheme tends to a time discretization of the incompressible Navier Stokes equations. We obtain for $\epsilon \rightarrow 0$ the Poisson equation for the pressure

$$
\Delta p^{n+1}=\frac{1}{k} \operatorname{div} \mathbf{u}^{n}-\operatorname{div} \operatorname{div} \boldsymbol{\Theta}^{n},
$$

together with

$$
\begin{gathered}
\mathbf{u}^{n+1}=\mathbf{u}^{n}-k\left(\operatorname{div} \boldsymbol{\Theta}^{n}+\nabla p^{n+1}\right), \\
\boldsymbol{\Theta}^{n+1}=2 \tau \mathbf{S}\left[\mathbf{u}^{n+1}\right]-\mathbf{u}^{n+1} \otimes \mathbf{u}^{n+1} .
\end{gathered}
$$

Thus, in the limit, we have obtained the usual projection method for the incompressible Navier-Stokes equations; see, e.g. [39]. We note that the incompressibility condition is fulfilled for $\mathbf{u}$ in every time step.

In IMEX notation the above first order scheme is given by the explicit and implicit Butcher tableau [4, 38]

$$
\begin{array}{l|lll|ll}
0 & 0 & 0 \\
1 & 1 & 0 \\
\hline & 1 & 0
\end{array} \quad \begin{array}{llll}
0 & 1 & 0 & 1 \\
\hline
\end{array} \quad \begin{array}{lll}
0 & 0 & 1
\end{array}
$$


For the above semi-implicit time discretization the usual hyperbolic and parabolic CFL conditions have to be fulfilled to guarantee stability.

For the second order time discretization we choose a two stage IMEX Runge Kutta method [38, 4, which guarantees second order accuracy in the stiff limit. The associated explicit and implicit Butcher tableau are

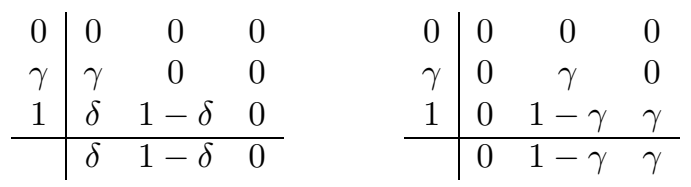

with $\gamma=1-\sqrt{2} / 2$ and $\delta=1-1 / 2 \gamma$. This yields

Step 1:

$$
\begin{aligned}
\Delta p^{n+1 / 2}-\frac{\epsilon^{2}}{k^{2} \gamma^{2}} p^{n+1 / 2}= & -\frac{\epsilon^{2}}{k^{2} \gamma^{2}} p^{n}+\frac{1}{k \gamma} \operatorname{div} \mathbf{u}^{n}-\operatorname{div} \operatorname{div} \boldsymbol{\Theta}^{n}, \\
\mathbf{u}^{n+1 / 2}= & \mathbf{u}^{n}-k \gamma\left(\operatorname{div} \boldsymbol{\Theta}^{n}+\nabla p^{n+1 / 2}\right), \\
\boldsymbol{\Theta}^{n+1 / 2}= & \boldsymbol{\Theta}^{n}-k \gamma \nabla^{\mathbf{a}}\left[\mathbf{u}^{n}\right] \\
& -\frac{k \gamma}{\epsilon^{2} \tau}\left(\boldsymbol{\Theta}^{n+1 / 2}-\mathbf{u}^{n+1 / 2} \otimes \mathbf{u}^{n+1 / 2}+2 \tau \mathbf{S}^{\epsilon}\left[\mathbf{u}^{n+1 / 2}\right]\right) .
\end{aligned}
$$

Step 2:

$$
\begin{aligned}
\Delta p^{n+1}-\frac{\epsilon^{2}}{k^{2} \gamma^{2}} p^{n+1}= & -\frac{\epsilon^{2}}{k^{2} \gamma^{2}} p^{n}+\frac{1}{k \gamma}\left((1-\gamma) \operatorname{div} \mathbf{u}^{n+1 / 2}+\gamma \operatorname{div} \mathbf{u}^{n}\right) \\
& -\left(\delta \operatorname{div} \operatorname{div} \boldsymbol{\Theta}^{n}+(1-\delta) \operatorname{div} \operatorname{div} \boldsymbol{\Theta}^{n+1 / 2}\right)-\frac{1-\gamma}{\gamma} \Delta p^{n+1 / 2}, \\
\mathbf{u}^{n+1}= & \mathbf{u}^{n}-k\left(\delta \operatorname{div} \boldsymbol{\Theta}^{n}+(1-\delta) \operatorname{div} \boldsymbol{\Theta}^{n+1 / 2}\right) \\
& -k\left((1-\gamma) \nabla p^{n+1 / 2}+\gamma \nabla p^{n+1}\right), \\
\boldsymbol{\Theta}^{n+1}= & \boldsymbol{\Theta}^{n}-k\left(\delta \nabla^{\mathbf{a}}\left[\mathbf{u}^{n}\right]+(1-\delta) \nabla^{a}\left[\mathbf{u}^{n+1 / 2}\right]\right) \\
& -\frac{k}{\epsilon^{2} \tau}\left((1-\gamma)\left(\boldsymbol{\Theta}^{n+1 / 2}-\mathbf{u}^{n+1 / 2} \otimes \mathbf{u}^{n+1 / 2}+2 \tau \mathbf{S}^{\epsilon}\left[\mathbf{u}^{n+1 / 2}\right]\right)\right. \\
+ & \left.\gamma\left(\boldsymbol{\Theta}^{n+1}-\mathbf{u}^{n+1} \otimes \mathbf{u}^{n+1}+2 \tau \mathbf{S}^{\epsilon}\left[\mathbf{u}^{n+1}\right]\right)\right) .
\end{aligned}
$$

For $\epsilon \rightarrow 0$ we obtain a second order time discretization of the INS equations based on the explicit scheme in the above IMEX method. Note that the divergence free condition is guaranteed in the limit in every time step.

A third order method is developed based on a third order IMEX scheme [4, 38. The associated explicit and implicit tables are 


\begin{tabular}{c|ccccc}
0 & 0 & 0 & 0 & 0 & 0 \\
$\frac{1}{2}$ & $\frac{1}{2}$ & 0 & 0 & 0 & 0 \\
$\frac{2}{3}$ & $\frac{11}{18}$ & $\frac{1}{18}$ & 0 & 0 & 0 \\
$\frac{1}{2}$ & $\frac{5}{6}$ & $-\frac{5}{6}$ & $\frac{1}{2}$ & 0 & 0 \\
1 & $\frac{1}{4}$ & $\frac{7}{4}$ & $\frac{3}{4}$ & $-\frac{7}{4}$ & 0 \\
\hline & $\frac{1}{4}$ & $\frac{7}{4}$ & $\frac{3}{4}$ & $-\frac{7}{4}$ & 0
\end{tabular}

\begin{tabular}{c|ccccc}
0 & 0 & 0 & 0 & 0 & 0 \\
$\frac{1}{2}$ & 0 & $\frac{1}{2}$ & 0 & 0 & 0 \\
$\frac{2}{3}$ & 0 & $\frac{1}{6}$ & $\frac{1}{2}$ & 0 & 0 \\
$\frac{1}{2}$ & 0 & $-\frac{1}{2}$ & $\frac{1}{2}$ & $\frac{1}{2}$ & 0 \\
1 & 0 & $\frac{3}{2}$ & $-\frac{3}{2}$ & $\frac{1}{2}$ & $\frac{1}{2}$ \\
\hline & 0 & $\frac{3}{2}$ & $-\frac{3}{2}$ & $\frac{1}{2}$ & $\frac{1}{2}$
\end{tabular}

The scheme was selected based on the fact that it seemed to have reasonable convergence and stability properties based on the results presented in [38. We omit for brevity the details of the scheme.

Remark 2. In the previous schemes it is of paramount importance that the implicit part of the time integrator is diagonally implicit. Fully implicit schemes (for which the Butcher tableau of the implicit part contains nonzero elements above the main diagonal) originate systems of nonlinear algebraic equations that need to be solved using suitable iterative techniques.

Remark 3. In order to develop a relaxed scheme for the limit INS equations only, one may use the simplified relaxation system (13).

\section{Numerical RESUlts AND EXAMPLES}

In this section we test the above schemes in several different situations. Our test examples can be considered in four general categories: starting with the 1-D time dependent problem we first perform an accuracy test for the third order scheme; second, we test if the scheme converges uniformly to the limit $\epsilon \rightarrow 0$; in part 2 we consider a stationary problem in 2-D and test the ability of our scheme to resolve discontinuous, solutions especially for the convection terms; and in part 3 the scheme is tested on time dependent incompressible Navier-Stokes problems in 2-D. Indeed with such a simplified approach, it will be demonstrated that competitive schemes for solving incompressible flow problems have been developed.

4.1. Accuracy and convergence for 1-D test problems. Accuracy and convergence of the scheme developed in the previous section is numerically investigated for the relaxation system leading to the inviscid and viscous Burgers equation in one space dimension, respectively. That means we consider a system analogous to (13):

$$
\begin{aligned}
\partial_{t} u+\partial_{x} \Theta & =0 \\
\partial_{t} \Theta+a \partial_{x} u & =-\frac{1}{\epsilon^{2}}\left(\Theta-\frac{1}{2} u^{2}+\tau \partial_{x} u\right) .
\end{aligned}
$$

We consider the third order method developed above based on the CWENO reconstruction and the third order IMEX scheme.

\section{Test 1: Accuracy}

In order to check the accuracy of our third-order relaxation scheme, we first consider the one-dimensional inviscid Burgers equation $(\tau=0$ and $\epsilon=0)$. We solve the equations (20) in $[0,2 \pi]$ augmented with the smooth initial data, $u(x, 0)=0.5+$ $\sin (x)$, and periodic boundary conditions. We discretize the spatial domain into $N$ gridpoints, and we choose the relaxation parameter $a=1.5$ in all computations. Recall that the unique entropy solution of (20) is smooth up to the critical time 
$T=1$. In Table 1 we show the error norms at the pre-shock time $t=0.5$ when the solution is still smooth using CFL $=0.75$. The errors are measured by the difference between the pointvalues of the exact solution and the reconstructed pointvalues of the computed solution. As expected our scheme preserves the third order of accuracy.

TABLE 1. Error-norms for the invscid Burgers problem.

\begin{tabular}{ccccccc}
\hline$N$ & $L^{\infty}$-error & Rate & $L^{1}$-error & Rate & $L^{2}$-error & Rate \\
\hline 40 & $0.37681 \mathrm{E}-01$ & - & $0.28977 \mathrm{E}-01$ & - & $0.30533 \mathrm{E}-01$ & - \\
80 & $0.15964 \mathrm{E}-01$ & 1.239 & $0.71792 \mathrm{E}-02$ & 2.013 & $0.82323 \mathrm{E}-02$ & 1.891 \\
160 & $0.47363 \mathrm{E}-02$ & 1.753 & $0.12559 \mathrm{E}-02$ & 2.515 & $0.17511 \mathrm{E}-02$ & 2.233 \\
320 & $0.78772 \mathrm{E}-03$ & 2.588 & $0.14477 \mathrm{E}-03$ & 3.117 & $0.22551 \mathrm{E}-03$ & 2.957 \\
640 & $0.69819 \mathrm{E}-04$ & 3.496 & $0.92831 \mathrm{E}-05$ & 3.963 & $0.17196 \mathrm{E}-04$ & 3.713 \\
1280 & $0.65638 \mathrm{E}-05$ & 3.411 & $0.61968 \mathrm{E}-06$ & 3.905 & $0.13613 \mathrm{E}-05$ & 3.659 \\
\hline
\end{tabular}

\section{Test 2: Uniform Convergence}

In this example we investigate the uniform convergence behavior of the relaxing method for different values of $\epsilon$. The uniform convergence of the method is numerically investigated for the relaxation system, equation (20), leading to the one-dimensional viscous Burgers equation. Once again we consider the third order method developed above based on the CWENO reconstruction and the third order IMEX scheme.

We plot the convergence rates for different values of $\epsilon$ in Figure 1. The rates are determined by comparing the errors at time $T=1$ computed from $\Delta t=0.01$, $\Delta t=0.005, \Delta t=0.0025$, and $\Delta t=0.00125$, respectively.

In space the energy norm is used. Third order accuracy is reached for very small and very large values of $\epsilon$, whereas for intermediate values a slight deterioration of the accuracy is observed. This is expected and in good agreement with the results obtained in 38.
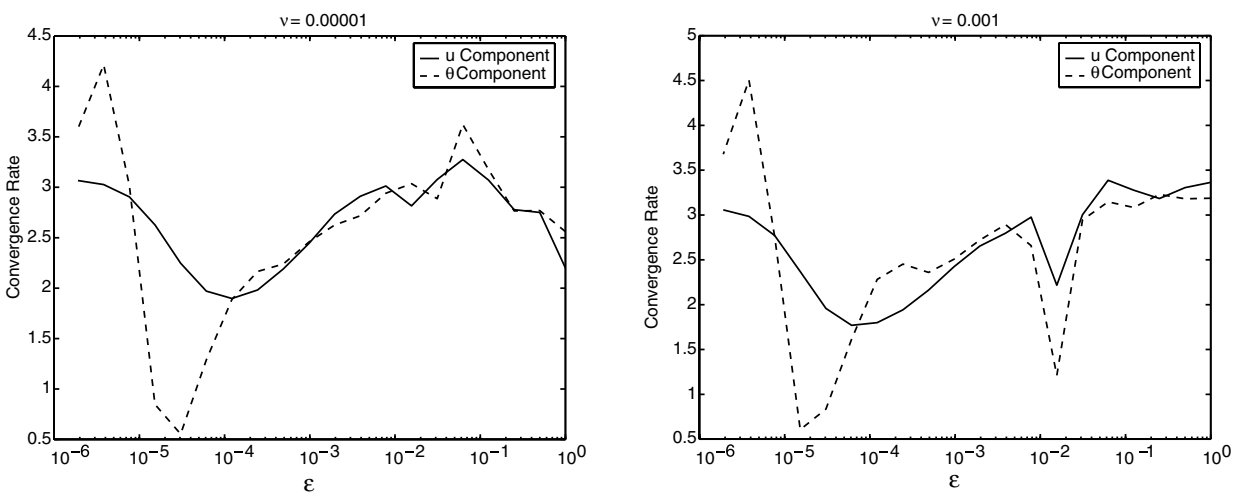

FiguRE 1. Convergence rates for $\tau=0.00001$ and $\tau=0.001$ 

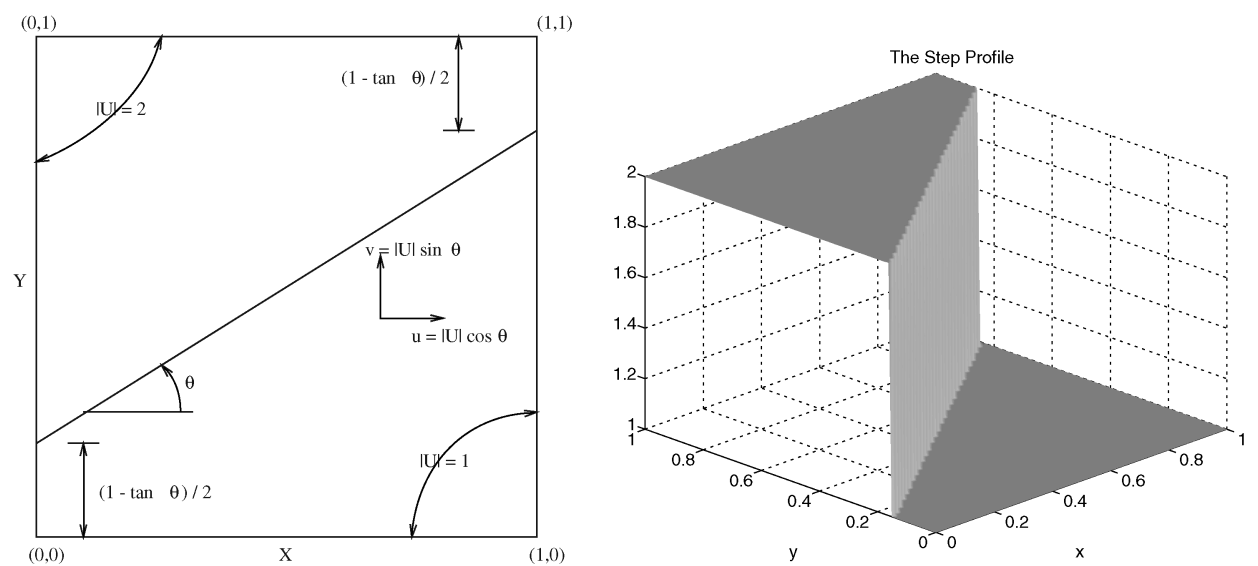

Figure 2. The setup of the step profile. The $z$-axis depicts $\|\mathbf{u}\|$.

4.2. Stationary 2-D test problems. The first problem is a simple test case to compare the spatial discretization of the convective term with the discretization given by other methods. We compare the second and third order relaxed schemes with different central methods [34, 31. The problem we consider is the stationary convection-diffusion problem [16]

$$
\operatorname{div}(\mathbf{u} \otimes \mathbf{u})=\tau \Delta \mathbf{u}
$$

on $[0,1]^{2}$. Here we consider the relaxed schemes $\epsilon \rightarrow 0$. For these stationary problems our main focus will be on the qualitative behaviour of our schemes to resolve the solutions with very sharp gradients.

The first example is pure convection of a step profile [16. More details on how this system is discretized and treated numerically can be found in [16]. This is a simple but good test problem for examining the relative performance of different numerical approximations to convection terms with sharp gradients for $u_{1}$ and $u_{2}$. We consider $(x, y)$ in $[0,1]^{2}$. The computation domain is divided into two subdomains which give a step profile as sketched in Figure 2.

The flow profile inside the domains is extended to the boundary. The computational domain is discretized using a $41 \times 41$ regular mesh for different flow angles $\theta$. Values for $\tau$ were also varied. The resulting nonlinear system is solved by the Newton method using a GMRES-based solver described and implemented in [27.

The results are plotted in Figure 3 . They show the computed profile at the line $x=\frac{1}{2}$ for both the velocity components $u_{1}$ and $u_{2}$. In the figures we make a comparison of the following second and third order schemes: Kurganov and Tadmor's (KT) second order scheme [34; Kurganov and Levy's (KL) third order scheme 11; the relaxed scheme based on Jin and Xin's (JX) second order approach [26] and the new third order relaxed scheme (RKL) based on CWENO reconstruction.

In this and the following tests we have used the local characteristic speeds as in [34, 1] to define $\mathbf{a}$ at the point $\left(x_{i}, y_{j}\right)$ :

$$
\begin{aligned}
a_{i+\frac{1}{2} j} & =2 \max \left\{\left|p_{i j}\left(u_{1} ; x_{i+\frac{1}{2}}\right)\right|,\left|p_{i+1 j}\left(u_{1} ; x_{i+\frac{1}{2}}\right)\right|\right\}, \\
b_{i j+\frac{1}{2}} & =2 \max \left\{\left|p_{i j}\left(u_{2} ; y_{j+\frac{1}{2}}\right)\right|,\left|p_{i j+1}\left(u_{2} ; y_{j+\frac{1}{2}}\right)\right|\right\},
\end{aligned}
$$



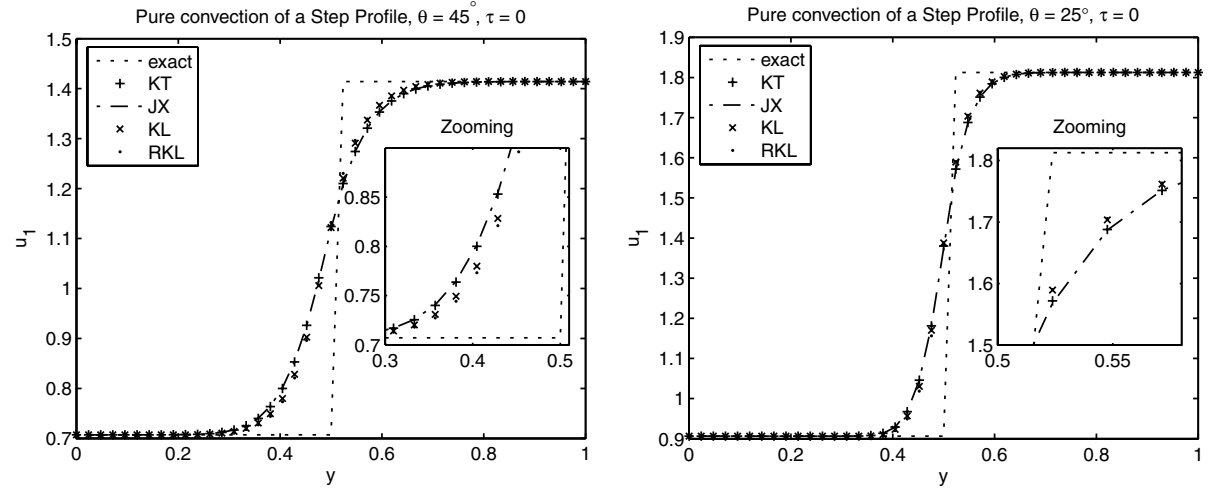

Figure 3. Comparison of different approximations of a stationary step profile for $u_{1}$ with different angles $\theta=\pi / 4,25$ degrees on the left and right, respectively.
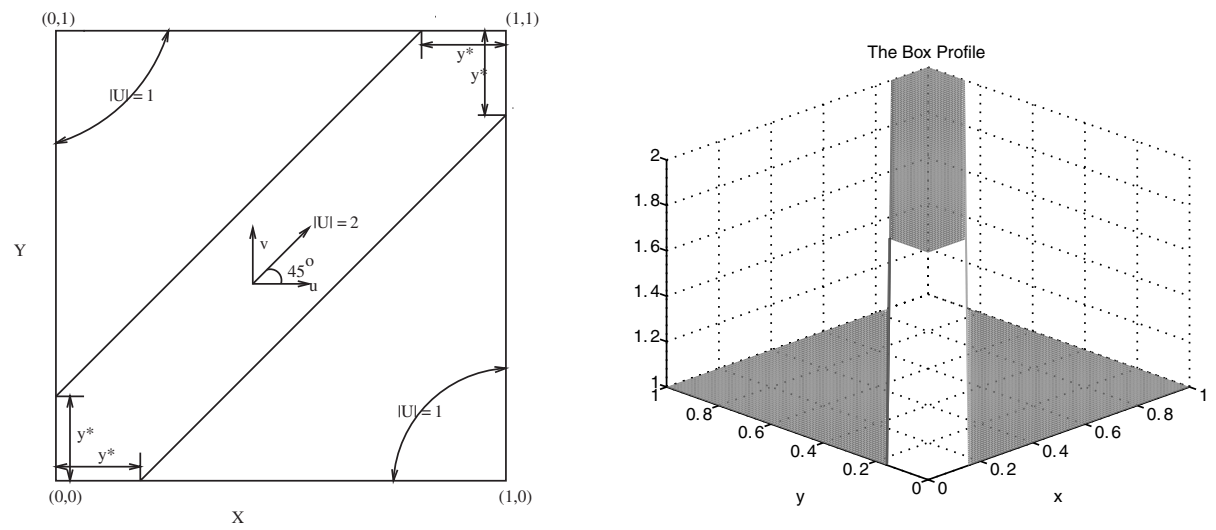

Figure 4 . The setup of the box profile. The $z$-axis depicts $\|\mathbf{u}\|$.
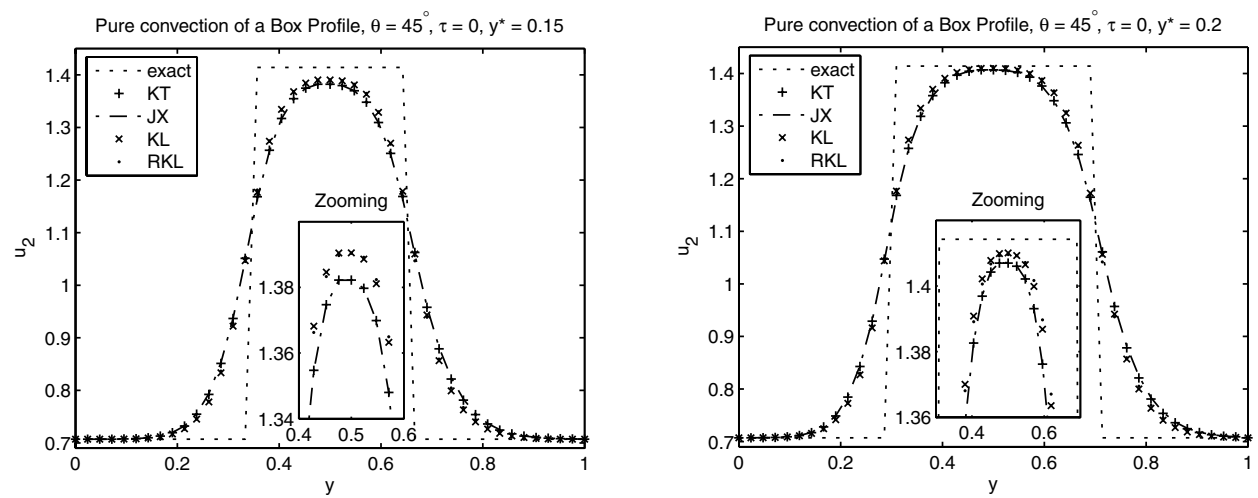

Figure 5. Comparison of different approximations for convection in stationary flow with a box profile. 
and $a_{i-\frac{1}{2} j}, b_{i j-\frac{1}{2}}$ in the same way. Here the polynomials $p_{i j}$ are defined for scalar variables $u_{1}$ and $u_{2}$ analogous to the definition in (15) or (16). The results for the step profile in Figure 2 are shown in Figure 3.

In this figure profiles for the $u_{1}$ are shown for $\tau=0$ and are compared with the exact stationary profile [16]. The profiles for $u_{2}$ are similar. Tests on small values of $\tau=10^{-6}$ were made, and the results are also similar. The second example is pure convection of a box profile. We consider a box-shaped profile as shown in Figure 4. This example was also presented in [16]. It is normally selected because the severe, rapid change in the gradients in velocity resembles, in different ways, many similar profiles found, for example, in practical flows in which severe peak profiles are characteristic across shear layers. As in the previous example we compare approximations of the profile across a vertical plane in the middle of the solution domain. We compare the different schemes using a uniform $41 \times 41$ mesh, and the results are presented in Figure 5.

In Figure 5, we present results for stationary profiles of $u_{2}$. The results for $u_{1}$ are very similar.

Here too, since we are mainly investigating the performance of the approximation on the convection terms, results for $\tau=0$ are presented. As one observes from the figures the second and third order relaxed methods give results that are qualitatively similar to the KT and KL scheme, respectively. In some cases, the relaxed scheme is more accurate, and in other cases RKL is more accurate.

4.3. Instationary 2-D incompressible Navier-Stokes test problems. Here we are especially interested in the results of the relaxing method for very small $\epsilon$ and the results of the relaxed method setting $\epsilon=0$ for the incompressible Navier-Stokes equations.

Problem 1: (Shear layer). The next problem is set up to test the behavior of the discretization for nonstationary situations when steep gradients are involved. We consider the following periodic problem [1]. Let $(x, y) \in[0,2 \pi]^{2}$. The initial conditions are

$$
u(x, y, 0)= \begin{cases}\tanh \left(\frac{1}{\rho}(y-\pi / 2)\right), & y \leq \pi \\ \tanh \left(\frac{1}{\rho}(3 \pi / 2-y)\right), & y>\pi\end{cases}
$$

and

$$
v(x, y, 0)=\delta \sin (x)
$$

with $\delta=0.05$ and $\rho=\pi / 15$. We use $64 \times 64$ and $128 \times 128$ spatial grid points, respectively. The vorticity system (14) and the associated relaxing method is used.

First we consider the situation in the incompressible Navier Stokes/Euler limit. We compute the solution using the relaxing scheme with $\epsilon=10^{-6}$ combined with an IMEX method of first, second and third order. 
Figure 6 shows the evolution at time $t=4$ for the first, second and third order methods described in the paper for the Euler case $(\tau=0$ in (14) $)$ and $\epsilon=10^{-6}$. Figure 7 shows the evolution at time $t=10$ for the first, second and third order methods for the Navier-Stokes case with $\tau=0.01$ and $\epsilon=10^{-6}$.

To compare these results qualitatively with other methods, see for example 1]. A closer convergence study for the vorticity variable yields the results displayed in Tables 2 and 3 The results show the second and third order of the schemes, respectively, in the shear layer case. In the second order case we have used a van Leer limiter instead of the minmod limiter. Minmod gives slightly worse results. As a reference solution we used the solution obtained on the finest mesh of $528 \times 528$ gridpoints.

Finally, the situation with large $\epsilon$ is considered. Figure 8 shows the evolution at time $t=10$ for the third order methods for the Navier-Stokes case with $\tau=0.01$ and $\epsilon=0.1$ and for comparison $\epsilon=10^{-6}$.

TABLE 2. Error-norms for the double shear layer problem with $\tau=0$ and $\epsilon=10^{-6}$ at $t=2$.

\begin{tabular}{ccccccc}
\hline Gridpoints & $L^{\infty}$-error & Rate & $L^{1}$-error & Rate & $L^{2}$-error & Rate \\
\hline $16 \times 16$ & $3.15715 \mathrm{E}-01$ & - & $5.79035 \mathrm{E}-01$ & - & $4.26853 \mathrm{E}-01$ & - \\
$32 \times 32$ & $8.54186 \mathrm{E}-02$ & 1.886 & $1.79334 \mathrm{E}-01$ & 1.691 & $1.26904 \mathrm{E}-01$ & 1.750 \\
$64 \times 64$ & $2.23542 \mathrm{E}-02$ & 1.934 & $4.91291 \mathrm{E}-02$ & 1.868 & $3.39324 \mathrm{E}-02$ & 1.903 \\
$128 \times 128$ & $5.58856 \mathrm{E}-03$ & 2.000 & $1.26978 \mathrm{E}-02$ & 1.952 & $8.50078 \mathrm{E}-03$ & 1.997 \\
$264 \times 264$ & $1.29906 \mathrm{E}-03$ & 2.103 & $3.10696 \mathrm{E}-03$ & 2.031 & $1.98562 \mathrm{E}-03$ & 2.098 \\
\hline
\end{tabular}

TABLE 3. Error-norms for the double shear layer problem with $\tau=0$ and $\epsilon=10^{-6}$ at $t=2$.

\begin{tabular}{ccccccc}
\hline Gridpoints & $L^{\infty}$-error & Rate & $L^{1}$-error & Rate & $L^{2}$-error & Rate \\
\hline $16 \times 16$ & $1.12035 \mathrm{E}-02$ & - & $1.87151 \mathrm{E}-02$ & - & $1.62533 \mathrm{E}-02$ & - \\
$32 \times 32$ & $1.62774 \mathrm{E}-03$ & 2.783 & $2.88411 \mathrm{E}-03$ & 2.698 & $2.45659 \mathrm{E}-03$ & 2.726 \\
$64 \times 64$ & $2.16565 \mathrm{E}-04$ & 2.910 & $4.00016 \mathrm{E}-04$ & 2.850 & $3.35331 \mathrm{E}-04$ & 2.873 \\
$128 \times 128$ & $2.74676 \mathrm{E}-05$ & 2.979 & $5.23789 \mathrm{E}-05$ & 2.933 & $4.33645 \mathrm{E}-05$ & 2.951 \\
$264 \times 264$ & $3.42870 \mathrm{E}-06$ & 3.002 & $6.54736 \mathrm{E}-06$ & 3.000 & $5.42057 \mathrm{E}-06$ & 3.000 \\
\hline
\end{tabular}



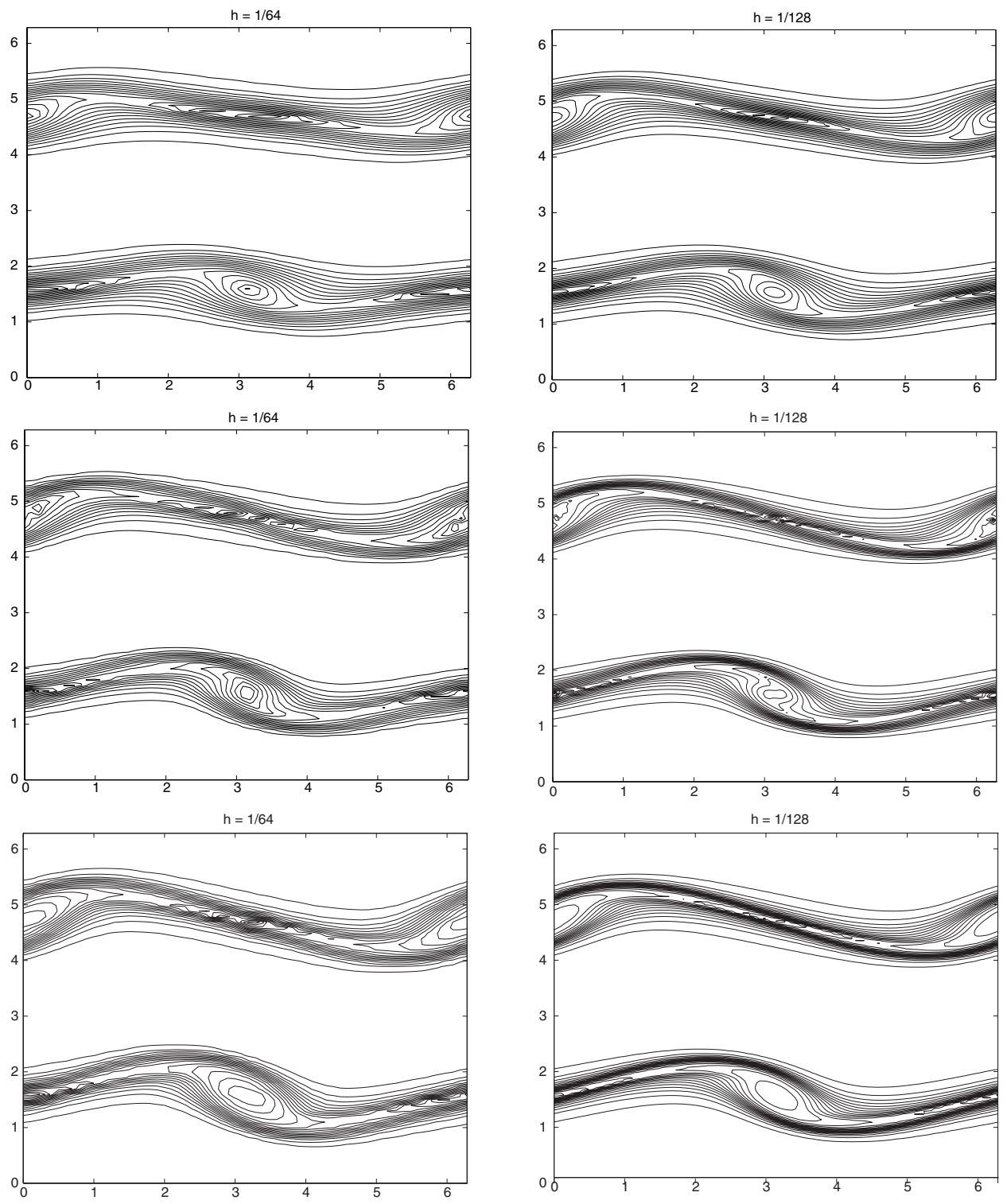

Figure 6. Results for the Euler case: first order (top), second order (medium) and third order (bottom). 

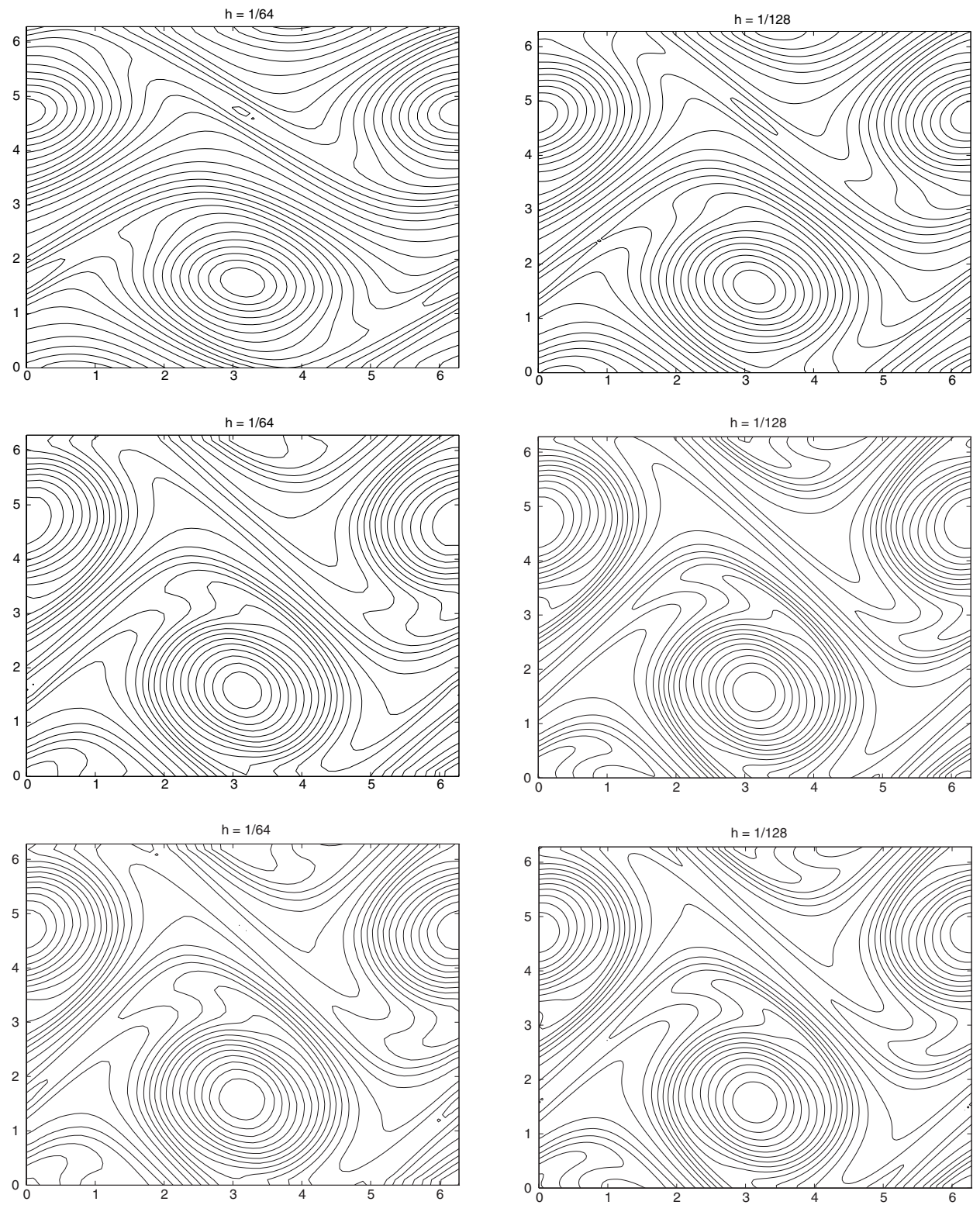

Figure 7. Results for the Navier-Stokes case: first order (top), second order (medium) and third order (bottom). 

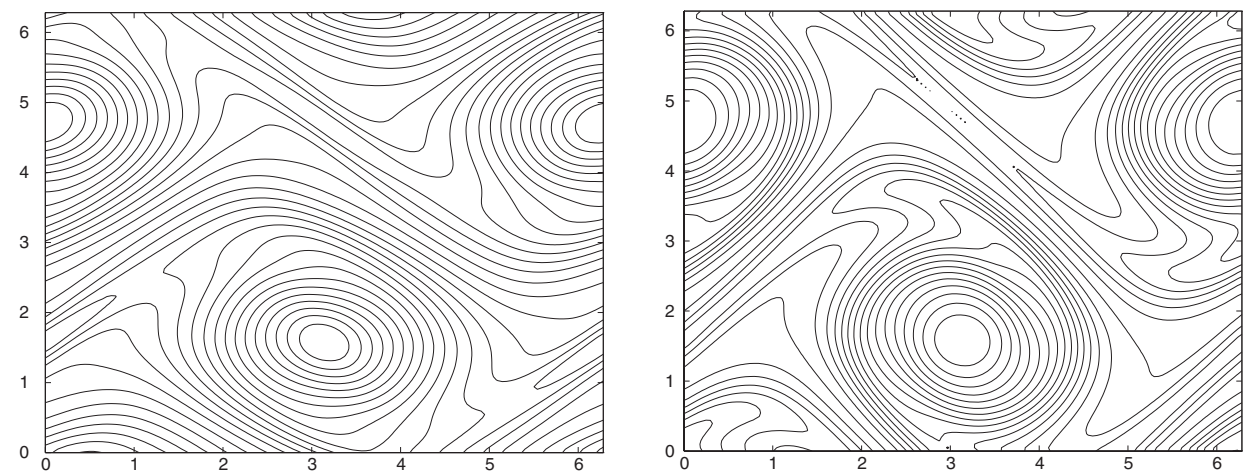

FiguRe 8. Results for the Navier-Stokes case: third order, $\epsilon=0.1$ and $\epsilon=10^{-6}$.
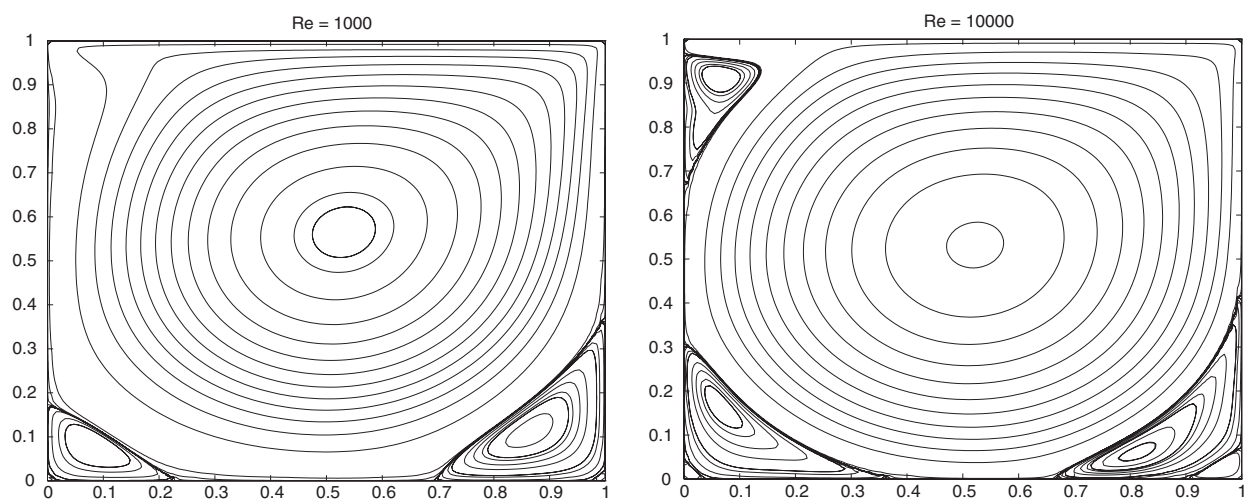

Figure 9. Driven Cavity, plot of the stream function for $R e=$ 1000 and $R e=10000$.

Problem 2: (Driven cavity). Finally, we consider a driven cavity situation with $\mathbf{x} \in[0,1]^{2}$ and the usual boundary conditions with a drift $\mathbf{u}=(\bar{u}, 0)$ parallel to the boundary at the top of the square and $\mathbf{u}=0$ at the other sides. For the simulation we use the third order relaxation method developed above based on the relaxation system (14). Zero initial conditions are used for all moments. $\bar{u}$ is chosen equal to $1 . \epsilon$ is again chosen equal to $10^{-6}$ for the relaxing scheme. We use $128 \times 128$ spatial grid points. The time step is chosen according to the CFL condition. Figure 9 shows a plot of the stream-functions for $R e=1000$ at $t=100$ and $R e=10000$ at $t=1000$.

\section{Conclusions}

1. Several relaxation systems based on a Lattice Boltzmann type discrete velocity model have been presented. In the diffusive limit the systems relax towards the incompressible Navier-Stokes equations.

2. Second and third order relaxation schemes working uniformly in the incompressible Navier-Stokes limit have been presented. 
3. The space discretization is obtained using second and third order upwind discretization based on slope limiters and CWENO discretizations.

4. For the time discretization high order IMEX Runge Kutta methods have been used to obtain uniform accuracy with respect to the stiff relaxation time [38.

\section{REFERENCES}

1. Kurganov A. and D. Levy. A third-order semi-discrete central scheme for conservation laws and convection diffusion equations. SIAM J. Sci. Comp., 22:1461-1488, 2000. MR 1797891 (2001j:65127)

2. D. Levy, G. Puppo and G. Russo. Central WENO schemes for hyperbolic systems of conservation laws. M2AN Math. Model. Numer. Anal., 33:547-571, 1999. MR1713238(2000f:65079)

3. D. Levy, G. Puppo and G. Russo. Compact central WENO schemes for multidimensional conservation laws. SIAM J. Sci. Comp., 22:656 - 672, 2000. MR1780619 (2001d:65110)

4. U. Ascher, S. Ruuth, and R. Spiteri. Implicit-explicit Runge-Kutta methods for timedependent partial differential equations. Appl. Numer. Math., 25:151-167, 1997. MR 1485812 (98i:65054)

5. C. Bardos, F. Golse, and D. Levermore. Fluid dynamic limits of kinetic equations: Formal derivations. J. Stat. Phys., 63:323-344, 1991. MR.1115587 (92d:82079)

6. R. Benzi, S. Succi, and M. Vergassola. The Lattice-Boltzmann equation: Theory and applications. Physics Reports, 222:145-197, 1992.

7. R.E. Caflisch, S. Jin, and G. Russo. Uniformly accurate schemes for hyperbolic systems with relaxation. SIAM J. Num. Anal., 34:246-281, 1997. MR1445737(98a:65112)

8. N. Cao, S. Chen, S. Jin, and D. Martinez. Physical symmetry and lattice symmetry in Lattice Boltzmann methods. Phys. Rev. E, 55:21, 1997.

9. H. Chen, S. Chen, and W. Matthaeus. Recovery of the Navier-Stokes equations using a Latticegas Boltzmann method. Physical Review A, 45:5339-5342, 1992.

10. S. Chen and G.D. Doolen. Lattice Boltzmann method for fluid flows. Ann. Rev. Fluid Mech., 30:329-364, 1998. MR.1609606 (98m:76118)

11. D. Aregba-Driollet, R. Natalini, and S.Q. Tang. Diffusive kinetic explicit schemes for nonlinear degenerate parabolic systems. Quaderno IAC 26, 2000.

12. A. De Masi, R. Esposito, and J.L. Lebowitz. Incompressible Navier Stokes and Euler limits of the Boltzmann equation. CPAM, 42:1189, 1989. MR1029125 (90m:35152)

13. D. d'Humières. Generalized Lattice-Boltzmann Equations in: AIAA Rarefied Gas Dynamics: Theory and Applications. Progress in Astronautics and Aeoronautics, 159:450-458, 1992.

14. G. Puppo F. Bianco and G. Russo. High order central schemes for hyperbolic system of conservation laws. SIAM J. Sci. Comput., 21:294-322, 1999. MR.1722134 (2000i:65118)

15. F. Liotta, V. Romano, and G. Russo. Central schemes for balance laws of relaxation type. SIAM J. Numer. Anal., 38:1337-1356, 2000. MR1790036(2001j:65128)

16. P.H. Gaskell and A.K.C. Lau. Curvature-compensated convective transport: Smart, a new boundedness-preserving transport algorithm. Int. J. Num. Meth. in Fluids, 8:617-641, 1988. MR 944575 (89c:76010)

17. L. Giraud, D. d'Humieres, and P. Lallemand. A lattice Boltzmann model for Jeffreys viscoelastic fluid. Europhys. Lett., 42:625-630, 1998.

18. G. Naldi, L. Pareschi, and G. Toscani. Relaxation schemes for PDEs and applications to second and fourth order degenerate diffusion problems. Surveys in Mathematics for Industry, 10:315 - 343, 2002. MR2012453 (2004i:65087)

19. X. He and L.S. Luo. A-priori derivation of the lattice Boltzmann equation. Phys. Rev. E, 55:6333-6336, 1997.

20. X. He and L.S. Luo. Lattice Boltzmann model for the incompressible Navier-Stokes equation. J. Stat. Phys., 88:927-944, 1997. MR1467637 (98g:82038)

21. T. Inamuro, M. Yoshino, and F. Ogino. Accuracy of the lattice Boltzmann method for small Knudsen number with finite Reynolds number. Phys. Fluids, 9:3535-3542, 1997. MR 1478126

22. G.S. Jiang and E. Tadmor. Non-oscillatory central schemes for multidimensional hyperbolic conservation laws. SIAM J. Sci. Comp., 19:1892, 1998. MR1638064 (99f:65128) 
23. S. Jin and D. Levermore. Numerical schemes for hyperbolic conservation laws with stiff relaxation terms. J. Comp. Phys., 126:449, 1996. MR1404381 (97g:65173)

24. S. Jin, L. Pareschi, and G. Toscani. Diffusive relaxation schemes for discrete-velocity kinetic equations. SIAM J. Num. Anal., 35:2405-2439, 1998. MR.1655853 (99k:76100)

25. S. Jin, L. Pareschi, and G. Toscani. Uniformly accurate diffusive relaxation schemes for multiscale transport equations. SIAM J. Numer. Anal., 35:2405-2439, 1999. MR 1655853 (99k:76100)

26. S. Jin and Z. Xin. The relaxation schemes for systems of conservation laws in arbitrary space dimensions. Comm. Pure Appl. Math., 48:235-276, 1995. MR.1322811 (96c:65134)

27. C.T. Kelley. Iterative methods for linear and nonlinear equations. SIAM, Philadelphia, 1995. MR:1344684 (96d:65002)

28. A. Klar. An asymptotic-induced scheme for nonstationary transport equations in the diffusive limit. SIAM J. Num. Anal., 35:1073-1094, 1998. MR.1619859 (99d:82063)

29. A. Klar. Relaxation schemes for a Lattice Boltzmann type discrete velocity model and numerical Navier Stokes limit. J. Comp. Phys., 148:1-17, 1999. MR1669711 (99i:76122)

30. R. Kupferman and E. Tadmor. A fast high-resolution second-order central scheme for incompresssible flows. Proc. Nat. Acad. Sci., 94:4848, 1997. MR.1453829 (98d:76119)

31. A. Kurganov, S. Noelle, and G. Petrova. Semi-discrete central-upwind scheme for hyperbolic conservation laws and Hamilton-Jacobi equations. SIAM J. Sci. Comp., 23:707 - 740, 2001. MR.1860961 (2003a:65065)

32. A.S. Almgren, J.B. Bell, and W.G. Szymczak. A numerical method for the incompressible Navier-Stokes equations based on approximate projection. SIAM J. Sci. Comp., 17:358 - 369, 1996. MR:1374285 (96j:76104)

33. J.B. Bell, P. Colella, and H.M. Glaz. A second order projection method for the incompressible Navier-Stokes equations. J. Comp. Phys., 85:257 - 283, 1989. MR.1029192 (90i:76002)

34. A. Kurganov and, E. Tadmor. New high-resolution central schemes for nonlinear conservation laws and convection-diffusion equations. J. Comp. Phys., 160:241-282, 2000. MR.1756766 (2001d:65135)

35. L. Pareschi. Central differencing based numerical schemes for hyperbolic conservation laws with relaxation terms. SIAM J. Num. Anal., 39:1395 - 1417, 2001. MR/1870848|(2002j:65086)

36. A. A. Medovikov. High order explicit methods for parabolic equations. BIT, 38:372, 1998. MR.1638136 (99i:65096)

37. H. Nessyahu and E. Tadmor. Non-oscillatory central differencing for hyperbolic conservation laws. J. Comp. Phys., 87:1505, 1990. MR1047564(91i:65157)

38. L. Pareschi and G. Russo. Implicit-explicit Runge-Kutta schemes for stiff systems of differential equations. In L. Brugnano and D. Trigiante, editors, Recent Trends in Numerical Analysis, pages $269-289,2000$.

39. R. Peyret and T. Taylor. Computational Methods for Fluid Flow. Springer Series in Computational Fluids, Berlin, 1983. MR681481 (84d:76004)

40. Y.H. Qian, D. d'Humieres, and P. Lallemand. Lattice BGK models for the Navier Stokes equation. Europhys. Letters, 17:479-484, 1992.

41. R. Sanders and A. Weiser. A high order staggered grid method for hyperbolic systems of conservation laws in one space dimension. Comp. Meth. in App. Mech. and Engrg., 75:91107, 1989. MR.1035749 (91e:65112)

42. X. Shan and X. He. Discretization of the Velocity Space in the Solution of the Boltzmann Equation. Phys. Rev. Letter, 80:65-68, 1998.

43. C.W. Shu and S. Osher. Efficient implementation of essentially non-oscillatory shock-capturing schemes. J. Comp. Phys., 126:202 - 228, 1996. MR1391627 (97e:65081)

44. Y. Sone. Asymptotic theory of a steady flow of a rarefied gas past bodies for small Knudsen numbers. In R. Gatignol and Soubbaramayer, editors, Advances in Kinetic Theory and Continuum Mechanics, Proceedings of a Symposium Held in Honour of Henri Cabannes (Paris), Springer, pages 19-31, 1990. 
School of Mathematical Sciences, University of KwaZulu-Natal, Private X01, 3209 Pietermaritzburg, South Africa

E-mail address: bandamk@ukzn.ac.za

Fachbereich Mathematik, TU Kaiserslautern, Erwin-Schroedinger-Str. 48, D-67663 Kaiserslautern, Germany

E-mail address: klar@mathematik.uni-kl.de

Department of Mathematics, University of Ferrara, Via Machiavelli 35, I-44100 FerRARA, ITALY

E-mail address: pareschi@dm.unife.it

Fachbereich Mathematik, TU Kaiserslautern, Erwin-Schroedinger-Str. 48, D-67663 Kaiserslautern, Germany

E-mail address: seaid@mathematik.uni-kl.de 